INRA Prod. Anim.,

2014, 27 (5), 337-346

\title{
Optimiser la composition corporelle et la qualité des viandes de volailles en modulant le métabolisme par les acides aminés alimentaires
}

\author{
S. TESSERAUD ${ }^{\prime}$, I. BOUVAREL ${ }^{2}$, P. FRAYSSE ${ }^{\prime}$, S. MÉTAYER-COUSTARD ${ }^{\prime}$, A. COLLIN ${ }^{1}$, M. LESSIRE ${ }^{l}$, C. BERRI \\ ${ }^{1}$ INRA, UR83 Recherches Avicoles, F-37380 Nouzilly, France \\ ${ }^{2}$ Institut Technique de l'Aviculture, Centre INRA Val de Loire, F-37380 Nouzilly, France \\ Courriel : Sophie.Tesseraud@tours.inra.fr
}

Pour optimiser la composition corporelle des volailles et la qualité des viandes tout en garantissant l'efficacité des systèmes de production, il faut comprendre et maîtriser les mécanismes régulant l'utilisation métabolique des nutriments. Les acides aminés figurent parmi les facteurs majeurs contrôlant le métabolisme. Ils sont à la fois les unités de construction des protéines, des substrats pour la synthèse de composés d'importance physiologique majeure (hormones, antioxydants...) et des régulateurs de l'expression de gènes et de cascades de signalisation intracellulaires.

Tout changement quantitatif (niveaux d'énergie, de protéines...) ou qualitatif (composition du régime, dynamique d'apport des nutriments...) dans l'alimentation des animaux affecte le métabolisme. Ceci a des conséquences sur la dépense énergétique et l'efficacité alimentaire, la répartition des nutriments entre et au sein des tissus et organes et donc, in fine, sur la composition corporelle et la qualité de la viande. Les études récentes en nutrition visent ainsi à manipuler le métabolisme pour optimiser la croissance musculaire et la qualité des viandes, tout en garantissant l'efficacité du système. Ceci nécessite d'identifier des verrous métaboliques sur lesquels il sera possible d'agir pour moduler le développement et les caractéristiques tissulaires des animaux, et proposer à terme des solutions innovantes et durables adaptées aux objectifs de production de viande.

Dans cet article de synthèse, nous présenterons dans une première partie les bases concernant la régulation nutritionnelle du métabolisme. Quelques exemples choisis et représentatifs seront utilisés pour illustrer ce type de recherches en privilégiant les résultats obtenus lors d'études récentes conduites chez les oiseaux. En termes d'effecteurs, les exemples présentés concernent en particulier la nature des nutriments (en privilégiant les acides aminés, encadré 1) et leur dynamique d'apport. Ils portent sur les différentes voies métaboliques (métabolismes protéique, énergétique et/ou glucidique) qui sont toutes étroitement connectées, avec des acteurs, des régulateurs et/ou des tissus cibles communs. Nous présenterons ensuite l'incidence des modifications du métabolisme par la nutrition sur la qualité des produits et, plus généralement, l'efficacité des systèmes de production de viande de volaille, au travers d'études impliquant des recherches fondamentales et plus appliquées.

\section{1 / Régulation nutritionnelle du métabolisme}

Les facteurs alimentaires ont des effets multiples sur le métabolisme protéinoénergétique, classiquement divisés en effets à court terme et effets à long terme, spécifiques du régime alimentaire et faisant intervenir la composition et l'abon- dance des nutriments. Cette revue ne visant pas l'exhaustivité, mais voulant poser les bases de compréhension des mécanismes souvent complexes de régulation nutritionnelle, nous présenterons ici l'effet des apports quantitatifs et qualitatifs en nutriments en nous limitant aux protéines et acides aminés. Par ailleurs, nous ne parlerons que peu ici des variations à court terme liées à la distribution du repas, très étudiées chez les mammifères (voir les revues de Reeds et Fuller 1983, Millward et al 1996, Dardevet et al 2012). En effet, le poulet, particulièrement quand il est jeune, consomme généralement son aliment tout au long de la journée pendant la phase d'éclairement. L'apport de nutriments au cours de la journée est donc relativement continu avec un état pseudo-stationnaire du métabolisme (Muramatsu 1990). Nous détaillerons en

Encadré 1. Fonctions des acides aminés (cf. tableaux 1 et 2).

Les acides aminés sont tout d'abord des substrats, notamment en premier de la synthèse des protéines. Ils sont aussi donneurs de groupements méthyle, précurseurs de monoxyde d'azote (NO), de divers métabolites ou composés d'importance biologique majeure (nucléotides puriques et pyrimidiques, carnitine, antioxydants comme le glutathion, la taurine et la créatine...), d'hormones telles que les hormones thyroïdiennes, de médiateurs et neurotransmetteurs (histamine, sérotonine, dopamine...). Ils sont utilisés en tant que substrats énergétiques via la néoglucogenèse et la cétogenèse. Enfin, ils agissent en tant que régulateurs de la sécrétion d'hormones (comme l'insuline), de l'expression de gènes et de la signalisation cellulaire (par exemple les voies GCN2 et mTOR régulant la traduction des ARNm en protéines). Ils jouent ainsi un rôle essentiel dans la régulation du métabolisme, de la croissance et du développement, de la réponse immunitaire et plus largement de la santé des animaux. 


\section{Encadré 2. Chrononutrition et rythme journalier d'apport des nutriments.}

On parle en général de chrononutrition lorsque l'on considère les rythmes des prises alimentaires, en particulier chez l'Homme. Cette terminologie a été utilisée pour l'apport des protéines (i.e. chrononutrition protéique) dans le cadre de stratégies pour lutter contre la dénutrition et la sarcopénie chez le sujet âgé. En effet, chez la personne âgée, la quantité minimale en acides aminés, nécessaire pour stimuler l'anabolisme suite à un repas, serait augmentée (Dardevet et al 2012). Dans le cadre de la chrononutrition protéique, il s'agit alors d'apporter $75-80 \%$ de l'apport protéique journalier sur un seul repas (régime protéique pulsé) pour améliorer la biodisponibilité des acides aminés et favoriser l'anabolisme protéique postprandial (Arnal et al 1999, 2002 ; Bouillanne et al 2014). Dans le même ordre d'idée, la cinétique de digestion des protéines et la vitesse d'absorption intestinale des acides aminés qui en résulte, ont également un effet majeur sur l'anabolisme protéique (Boirie et al 1997). II existe ainsi des protéines dites rapides (e.g. protéines du lactosérum) car rapidement libérées par l'estomac, puis hydrolysées dans le duodénum. A l'inverse, les protéines dites lentes (e.g. protéines des caséines) car elles précipitent dans l'estomac, sont libérées lentement dans l'intestin grêle et sont absorbées de manière prolongée sur plusieurs heures. Chez le sujet jeune, les protéines lentes permettent une meilleure rétention protéique alors que c'est l'inverse chez le sujet âgé (Boirie et al 1997, Gryson et al 2014). Par ailleurs, la synchronisation ou non de la disponibilité en nutriments (par exemple glucose et acides aminés) au sein de la journée peut aussi modifier le métabolisme, en particulier énergétique (e.g. modification de la production de chaleur et de l'énergie retenue sous forme de gras) chez le veau pré-ruminant (Van den Borne et al 2006a et $\mathrm{b}$; Van den Borne et al 2007). Ceci renvoie à des mécanismes plus ou moins complexes (Hocquette et al 2007) que nous ne détaillerons pas ici.

Encadré 3. Métabolisme des acides aminés chez les oiseaux, généralités et particularités.

Quelle que soit l'espèce animale, les produits de la digestion sont absorbés sous la forme d'acides aminés ou de petits peptides qui peuvent être rapidement hydrolysés. Les acides aminés libres sont directement utilisés par les organes à des fins d'anabolisme ou de catabolisme (synthèse protéique, catabolisme oxydatif...). Outre l'alimentation, ils peuvent provenir de la dégradation des protéines corporelles. L'ensemble de ces réactions communes aux différentes espèces forme ce que l'on appelle le métabolisme protéique. Alors que, chez les mammifères, l'urée constitue la principale forme d'élimination de l'azote, l'excrétion d'azote se fait sous forme d'acide urique chez les oiseaux qui ne possèdent pas l'enzyme permettant la première étape du cycle de l'urée. Ceci a des conséquences en termes de métabolisme et de nutrition chez les oiseaux, pour lesquels certains acides aminés sont « nutritionnellement indispensables » (cf. revues Wang et al 2013, Wu 2009 et Wu et al 2013) : i) l'arginine ne peut pas être synthétisée via le cycle de l'urée ; ii) la proline, synthétisée à partir de l'arginine, du glutamate ou de la glutamine chez la plupart des mammifères, ne peut pas l'être à des taux adéquats chez les oiseaux ; iii) la glycine, précurseur pour la synthèse d'acide urique, n'est pas synthétisée assez rapidement pour satisfaire les besoins métaboliques. Elle doit aussi être apportée en quantité suffisante dans le régime.

revanche les effets des variations d'apport dans le temps des nutriments (qu'on peut rapprocher d'une forme de « chrononutrition », encadré 2), avec des variations considérées ici sur une période supérieure à un jour.

\section{1 / Effet de l'apport en protéines et en acides aminés}

En alimentation, les acides aminés sont tout d'abord des unités de construction permettant la synthèse des protéines (tableau 1 ; encadré 3 ). Ils sont connus comme des facteurs anaboliques, qui induisent un gain protéique en stimulant la synthèse des protéines tout en inhibant leur dégradation. Ces effets sur le renouvellement des protéines (i.e. protéosynthèse et protéolyse) ont été clairement démontrés chez les mammifères et, pour ce qui nous concerne, plus spécifiquement chez les espèces aviaires (voir les revues de Muramatsu 1990, Tesseraud 1995, Tesseraud et al 2006, Hocquette et al 2007). Par exemple, les régimes déficients en protéines provoquent une inhibition de la synthèse des protéines, alors que l'effet sur la protéolyse semble dépendre de la gravité de la déficience.
L'action d'acides aminés particuliers sur le renouvellement protéique a été démontrée par la supplémentation de régimes déficients. Par exemple, chez le poulet, l'addition de méthionine (seule ou avec de l'arginine) dans un régime ne contenant pas de protéines augmente le taux de synthèse $($ en $\% / j)$ des protéines corporelles, et en particulier musculaires (Muramatsu et al 1985 et 1986). Ce résultat peut paraître surprenant puisqu'il n'y a pas d'acide aminé dans le régime si on excepte la méthionine et l'arginine. L'hypothèse avancée est une meilleure réutilisation des acides aminés endogènes, provenant de la protéolyse musculaire, ce qui améliore le bilan azoté et réduit la perte de poids chez les poulets carencés en protéines mais supplémentés en méthionine. En effet, les acides aminés issus de la protéolyse corporelle sont caractérisés par une relative déficience en méthionine, une part importante des acides aminés soufrés étant incorporée dans les plumes et donc non « mobilisable ». D'autres études ont été réalisées en faisant varier l'apport d'un acide aminé comme la méthionine ou la lysine dans un régime par ailleurs bien équilibré pour les autres acides aminés (Barnes et al 1995, Tesseraud et al 1996a et 2001). Ces conditions correspondent bien à la pratique où ces acides aminés industriels sont ajoutés aux régimes pour couvrir les besoins des animaux en limitant l'apport en protéines. La supplémentation en méthionine augmente ainsi la croissance musculaire, et en particulier l'accrétion et la synthèse de protéines dans les muscles gastrocnémien et pectoral des poulets (Barnes et al 1995). De même, la supplémentation en lysine d'un régime déficient en cet acide aminé, mais équilibré pour les autres, augmente les quantités de protéines synthétisées et déposées dans le muscle squelettique de poulet (Tesseraud et al 1996a et 2001). Il s'agit ici de l'illustration d'un concept essentiel en nutrition protéique : un seul de ces acides aminés apporté en quantité insuffisante dans l'alimentation (acide aminé « limitant » comme la lysine ou la méthionine) constituera un frein à la synthèse protéique. Les autres acides aminés, apportés en excès, seront catabolisés (catabolisme oxydatif) et pourront être utilisés à des fins énergétiques, avec pour corollaire une augmentation de l'excrétion azotée et un engraissement accru. Le but est ici de raisonner les apports alimentaires en acides aminés pour optimiser la croissance corporelle et tissulaire et limiter les rejets azotés, dans un contexte de réduction de l'apport protéique.

La masse musculaire dépend de l'équilibre entre la synthèse et la dégradation des protéines. Les études réalisées en utilisant des régimes avec un apport limité en lysine ont révélé la possibilité 
Tableau 1. Les Acides Aminés (AA), substrats et précurseurs de composés d'importance biologique majeure. Tableau synthétique conçu à partir des revues de Wu 2009 et 2013.

\begin{tabular}{|c|c|c|}
\hline Acides aminés & Produits & Fonctions touchées \\
\hline $\mathrm{AA}$ & $\begin{array}{l}\text { Unités de construction des protéines et } \\
\text { des peptides }\end{array}$ & Métabolisme protéique \\
\hline $\mathrm{AA}$ & Substrats énergétiques & Catabolisme oxydatif \\
\hline Gln, Glu, Asp & Substrats énergétiques (intestin) & Fonction intestinale \\
\hline Arg & Monoxyde d'azote (NO) & Métabolisme, pression sanguine \\
\hline $\begin{array}{l}\text { Lys, Met } \\
\text { Gly, Arg, Met }\end{array}$ & $\begin{array}{l}\text { Carnitine } \\
\text { Créatine }\end{array}$ & Métabolisme énergétique \\
\hline $\begin{array}{l}\text { Cys, Gly, Glu } \\
\text { His } \\
\text { Cys }\end{array}$ & $\begin{array}{l}\text { Glutathion } \\
\text { Carnosine } \\
\text { Taurine }\end{array}$ & Protection contre le stress oxydant \\
\hline $\begin{array}{l}\text { Glu } \\
\text { Trp } \\
\text { His } \\
\text { Tyr }\end{array}$ & $\begin{array}{l}\text { Acide } \gamma \text {-aminobutyrique GABA } \\
\text { Sérotonine } \\
\text { Histamine } \\
\text { Adrénaline/noradrénaline, dopamine }\end{array}$ & Neurotransmission \\
\hline Tyr & Hormones thyroïdiennes & Métabolisme \\
\hline $\begin{array}{l}\text { Gly, Asp, Gln, Ser } \\
\text { Gly }\end{array}$ & $\begin{array}{l}\text { Acides nucléiques, ATP } \\
\text { Acide urique }\end{array}$ & $\begin{array}{l}\text { Information génétique, Fonctions cellulaires } \\
\text { Excrétion d'azote }\end{array}$ \\
\hline
\end{tabular}

(1) Produit terminal de l'oxydation des acides aminés chez les oiseaux.

d'un effet majeur des niveaux alimentaires en cet acide aminé sur la protéolyse : l'intensité de la protéolyse dans le muscle pectoral de poulets en croissance est toujours plus élevée, avec des valeurs qui peuvent être doublées, chez les poulets carencés en lysine (carence d'environ $25 \%$ ), indépendamment de l'âge mais aussi du génotype (Tesseraud et al 1996a et 2001). Nous ne savons pas si cet effet particulièrement fort de la carence en lysine sur la protéolyse musculaire est spécifique à cet acide aminé ou non, et s'il dépend de l'espèce animale. Cependant, la carence en lysine diminue le dépôt protéique musculaire avec une réponse plus ou moins prononcée selon le génotype (Tesseraud et al 2001). De plus, la réponse dépend du muscle étudié, le muscle pectoral étant le plus affecté, avec des conséquences particulièrement importantes sur le rendement en filet (Tesseraud et al 1996c). Le type métabolique musculaire est certainement un facteur d'explication, sachant que la réponse du métabolisme protéique aux facteurs nutritionnels est généralement plus forte dans les muscles glycolytiques (comme le muscle pectoral de poulet) comparés aux muscles mixtes ou oxydatifs (Baillie et al 1991 chez le rat, Tesseraud et al 1996c chez le poulet). Une composante de la variabilité peut aussi être spécifique du muscle (i.e. reliée à sa position, fonction et sollicitation) (Baillie et al 1991).

Le concept de besoin en acides aminés vu en termes de quantité de substrat limitant, s'il est important en nutrition protéique (cf. paragraphes précédents), a été renouvelé depuis une dizaine d'années : les acides aminés ne sont plus uniquement considérés comme des substrats directs de la protéosynthèse mais aussi comme des médiateurs de voies métaboliques, de la même manière que certaines hormones (tableau 2). En effet, certains acides aminés agissent en tant que « nutriment-signal » en modulant l'activité de protéines kinases intracellulaires impliquées par exemple dans la régulation de la protéosynthèse et/ou de

\section{Encadré 4. Le stress oxydant.}

La notion de stress oxydant définit un état de déséquilibre entre la production d'espèces réactives de l'oxygène (ROS en anglais pour " reactive oxygen species ») et les défenses antioxydantes. Les espèces réactives de l'oxygène sont générées au niveau de la chaîne respiratoire mitochondriale. Dans des conditions normales, elles sont générées en faible quantité et jouent un rôle dans la signalisation cellulaire. Formées en trop grande quantité, elles vont conduire à des dommages oxydatifs, tels que des mutations au niveau de l'ADN, la peroxydation lipidique, la carbonylation des protéines. Pour lutter contre ces effets délétères, l'organisme dispose d'un ensemble complexe de défenses antioxydantes comprenant des enzymes (e.g. superoxyde dismutase, catalase, glutathion peroxydase) et des systèmes non enzymatiques impliquant par exemple les groupements thiols $(-\mathrm{SH})$ comme c'est le cas pour l'antioxydant majeur qu'est le glutathion. 
Tableau 2. Types de régulation potentiellement affectés par les Acides Aminés (AA) et fonctions majeures touchées. Tableau synthétique conçu à partir des revues de Wu 2009 et 2013, Wang et al 2012, ainsi que des travaux réalisés dans le domaine chez les oiseaux*.

\begin{tabular}{|c|c|c|c|c|}
\hline Types de régulation & Exemples & Acides aminés & Fonctions touchées & Références \\
\hline $\begin{array}{l}\text { Sécrétion } \\
\text { d'hormones }\end{array}$ & Insuline & Leu, Arg & Métabolisme & $\begin{array}{l}\text { Rideau et al (1986) } \\
\text { Rideau et al (1989) }\end{array}$ \\
\hline $\begin{array}{l}\text { Expression de } \\
\text { gènes }\end{array}$ & $\begin{array}{l}\text { Gènes reliés à la } \\
\text { protéolyse } \\
\text { Gènes myogéniques } \\
\text { Gènes de la lipogenèse } \\
\text { et oxydation } \\
\text { Mucines } \\
\text { Transporteurs }\end{array}$ & $\begin{array}{l}\text { Leu, Met, Arg } \\
\text { Met } \\
\text { Met, Arg } \\
\text { Thr } \\
\text { Leu, Ile, Lys }\end{array}$ & $\begin{array}{l}\text { Métabolisme protéique } \\
\text { Croissance du muscle } \\
\text { Métabolisme lipidique } \\
\text { Fonction intestinale } \\
\text { Transport de nutriments }\end{array}$ & $\begin{array}{l}\text { Tesseraud et al (2007) } \\
\text { Wen et al (2014) } \\
\text { Del Vesco et al (2013) } \\
\text { Fouad et al (2013) } \\
\text { Azzam et al (2011) } \\
\text { Humphrey et al (2006) }\end{array}$ \\
\hline $\begin{array}{l}\text { Signalisation } \\
\text { cellulaire }\end{array}$ & $\begin{array}{l}\text { Voie GCN2 } \\
\text { Voie mTOR } \\
\text { NO, H2S }\end{array}$ & $\begin{array}{l}\text { AA } \\
\text { Leu, Arg, Met } \\
\text { Lys, Met, Arg }\end{array}$ & $\begin{array}{l}\text { Métabolisme + } \\
\text { expression de gènes }+ \\
\text { fonctions cellulaires }\end{array}$ & $\begin{array}{l}\text { Métayer et al (2010) } \\
\text { Tesseraud et al (2007) }\end{array}$ \\
\hline Méthylation & $\begin{array}{l}\text { Méthylation de l'ADN } \\
\text { Méthylation de protéines }\end{array}$ & $\begin{array}{l}\text { Met, Gly, His, Ser } \\
\text { Lys, Met, Arg, His }\end{array}$ & $\begin{array}{l}\text { Métabolisme }+ \\
\text { expression de gènes }+ \\
\text { fonctions cellulaires }\end{array}$ & \\
\hline
\end{tabular}

Références de travaux réalisés chez les oiseaux, quand existantes.

Figure 1. Nombreux rôles joués par les acides aminés dans la régulation du métabolisme: 1a) substrats pour la synthèse protéique et 1b) utilisation à des fins énergétiques (catabolisme oxydatif), 2) nutriment signal, 3) fonction anti-oxydante, 4) donneurs de groupement méthyle. D’après la revue de Tesseraud et al (2011).

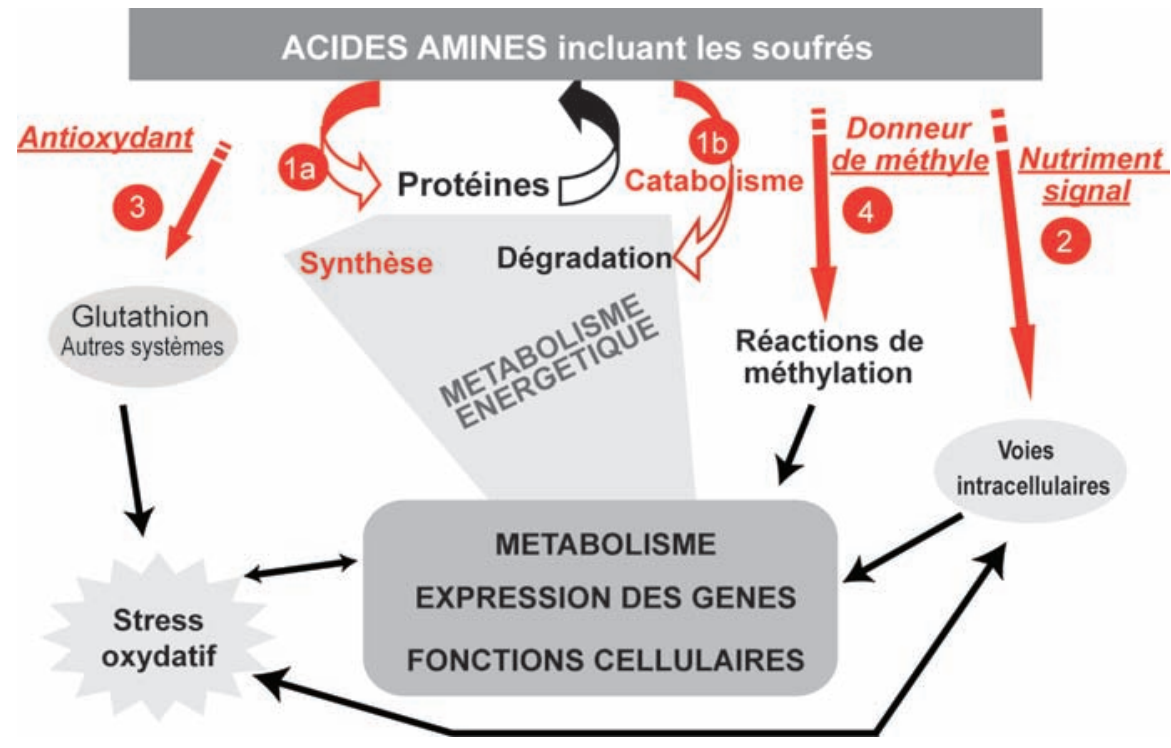

la protéolyse (voir revue de Tesseraud et al 2011, figure 1). Les acides aminés sont également les précurseurs de molécules importantes. Par exemple, la méthionine est un donneur de groupements méthyle, et joue de ce fait un rôle considérable sur les réactions de méthylation biologique, telle que la méthylation de l'ADN et des histones (protéines nucléaires associées à l'ADN), un processus qui influe nine est aussi un précurseur de la cystéine. La cystéine est nécessaire pour la synthèse du glutathion (i.e. tripeptide L-glutamylL-cysteinyl-glycine) et de la taurine, qui sont des composés essentiels de défense de l'hôte contre le stress oxydant (encadré 4) (Métayer et al 2008, Tesseraud et al 2009b). Cet ensemble de rôles joués par les acides aminés doit être pris en compte pour optimiser la nutrition. En effet, ils influencent le métabolisme protéinoénergétique, le stress oxydant, etc. et donc de nombreux paramètres en relation avec la qualité des produits (cf. $\S 2$ ).

\section{2 / Dynamique d'apport des nutriments}

La régulation nutritionnelle du métabolisme inclut les effets des quantités ingérées et de la composition du régime, mais dépend aussi du rythme d'apport des nutriments (Walrand et Boirie 2005, Hocquette et al 2007). Les travaux chez les mammifères intègrent généralement une régulation sur la journée (encadré 2). Chez le poulet, nous rapporterons des études conduites sur un pas de temps plus long en utilisant des stratégies d'alimentation séquentielle qui consistent à distribuer alternativement des aliments de composition ou caractéristiques différentes.

Dans un modèle d'alternance sur un cycle de 48 heures de régimes iso-énergé- 
tiques et iso-protéiques mais variant sur la teneur en un acide aminé, la lysine, Tesseraud et al (2009a) ont montré une régulation de la protéolyse musculaire et une forte capacité des poulets à s'adapter aux changements d'apport en cet acide aminé. En particulier, les variations quotidiennes de la teneur en lysine alimentaire affectent l'expression de deux gènes importants dans le contrôle de la protéolyse musculaire, i.e. $M u R F 1$ et Atrogin-1. Ces gènes sont ainsi surexprimés dans le muscle de poulet soumis à une carence transitoire en lysine. Dans une étude plus récente (Boussaid-Om Ezzine et al 2012), la régulation de ces mêmes gènes a été examinée dans un modèle d'alimentation séquentielle plus complexe, avec des alternances d'un jour sur l'autre de régimes plus ou moins riches en protéines et/ou en énergie. L'expression de MuRF1 et Atrogin-1 au niveau du muscle pectoral du poulet est fortement modifiée par les alternances de régimes, surtout lorsque l'on fait varier le taux protéique (ces gènes sont surexprimés avec la distribution d'un régime moins riche en protéines et à l'inverse sous-exprimés avec la distribution d'un régime plus riche en protéines). En parallèle, les voies de régulation de la protéosynthèse sont affectées (ces voies sont par exemple activées avec la distribution du régime enrichi en protéines). Il n'est pas possible de mesurer l'évolution de la masse protéique d'un muscle à l'échelle d'une journée. Cependant, nous pouvons supposer qu'il existe des évolutions au sein d'un cycle de 48 heures, avec un phénomène de «yoyo » au niveau du muscle pectoral en fonction des périodes de plus ou moins fort anabolisme. Même si ce phénomène existe, plusieurs questions restent en suspens : observerait-on la même réponse quel que soit le muscle étudié ? Est-ce généralisable à d'autres espèces que le poulet, ou même dépendant de l'intensité de croissance des animaux (valable par exemple chez les poulets à croissance rapide mais pas chez ceux à croissance lente) ? Quelles sont les limites de cette stratégie alimentaire ? Quel rôle joue le facteur temps, notamment la période et la cyclicité des apports, ou l'âge de l'animal (encadré 5)?

D'autres travaux sur le modèle d'alimentation séquentielle montrent par ailleurs une régulation à court terme des métabolismes énergétique et glucidique du poulet en fonction des alternances de la distribution de régimes riches en protéines et/ou en énergie. Dans ces travaux, il s'agissait, par exemple, d'explorer l'activation potentielle de voies de signalisation contrôlant l'utilisation du glucose par le muscle (le glucose est le substrat énergétique majeur utilisé par le muscle pectoral du poulet). A été ciblée en particulier la voie comprenant la protéine
Encadré 5. Le temps, notamment la période et la cyclicité des apports, et l'âge de l'animal, affectent les réponses du métabolisme.

Les phénomènes qui sont étudiés dans les études métaboliques dépendent clairement du temps. Ceci recouvre au moins deux notions : i) la durée de l'effet avec les classifications d'effets aigus ou chroniques ou, encore, effets à court, moyen ou long terme et ii) l'âge auquel le traitement est appliqué.

Dans le modèle d'alimentation séquentielle, la régulation testée serait plutôt dite à court terme puisque les études métaboliques sont réalisées quelques heures après le changement de l'aliment. Le fait que, dans cette stratégie alimentaire, des cycles soient utilisés implique également que ce qui est observé à un moment donné dépend de l'aliment consommé dans la phase précédente du cycle, par exemple le jour précédent pour des cycles de 48 heures. Les phénomènes sont un peu similaires pour l'ingestion : après plusieurs cycles de 48 heures, une éventuelle adaptation de l'ingestion (si elle existe) pourrait aussi dépendre de ce qui a été consommé la veille. L'étude des cinétiques sur plusieurs cycles et différents temps de prélèvements paraît nécessaire pour mieux appréhender les régulations sous-jacentes et tester une éventuelle adaptation nutritionnelle à plus long terme.

Chez le poulet comme chez les mammifères, le métabolisme musculaire et sa régulation dépendent de l'âge. Un des phénomènes les plus marquants est une réduction du taux de synthèse des protéines avec l'âge, plus ou moins marquée selon les tissus, mais aussi selon la période considérée (pour revue Tesseraud 1995, Tesseraud et al 1996b). De plus, l'effet de facteurs anaboliques ou de la stimulation postprandiale est beaucoup plus prononcé chez les très jeunes animaux, que l'on considère l'effet sur la synthèse protéique ou sur les voies de signalisation qui la contrôlent (Davis et al 2008). II semble donc important de prendre en compte l'âge de l'animal pour optimiser les stratégies alimentaires et/ou d'élevage.

Figure 2. Enzymes impliquées dans la régulation du métabolisme du glycogène musculaire: AMPK : "Adenosine Monophosphate-activated Protein Kinase »; PhK : Phosphorylase Kinase ; GS : Glycogène Synthase ; PYG : Glycogène Phosphorylase. D'après Sibut et al (2008), Wilson et al (2011).

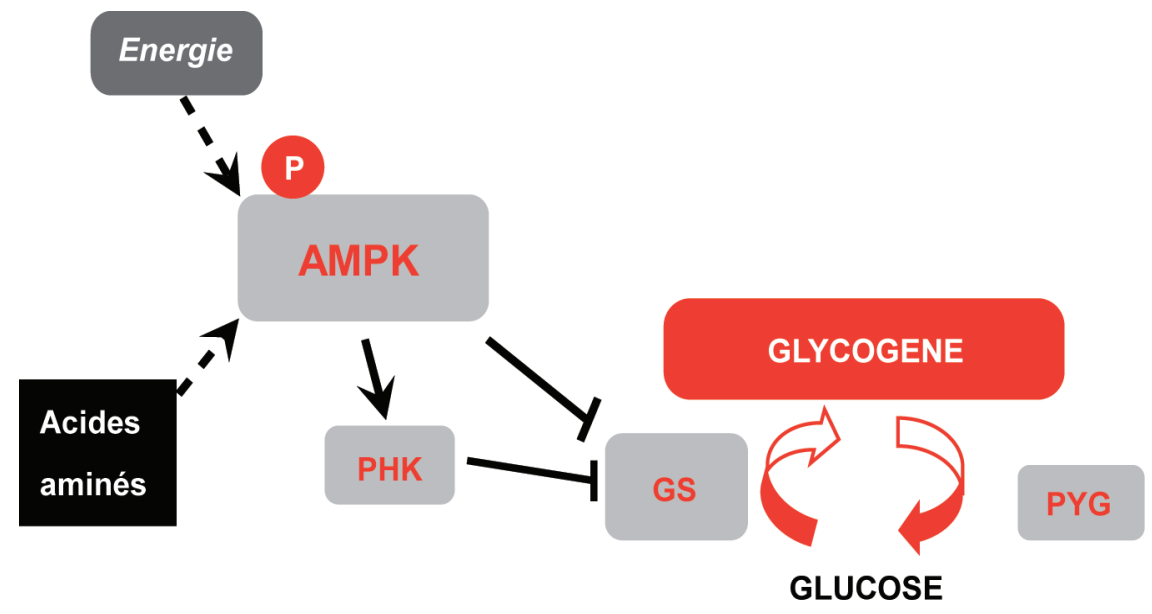

kinase, «AMP-activated protein kinase » (AMPK). L'AMPK est un senseur énergétique qui joue un rôle clé dans la régulation des métabolismes énergétique et glucidique chez le poulet comme chez les mammifères (ProszkowiecWeglarz et al 2006, Sibut et al 2008). Cette voie peut intervenir dans la régulation de la synthèse du glycogène, notamment en modifiant l'activité de la Glycogène Synthase (GS) (figure 2). Les résultats obtenus indiquent une régulation à court terme du métabolisme du glycogène musculaire du poulet, avec une implication particulière de l'AMPK (Mameri et al 2010). Il est intéressant de constater que ce ne sont pas les mêmes séquences d'alimentation qui induisent les activations des voies impliquées dans le contrôle du métabolisme protéique et du métabolisme glucidique. Par exemple, la voie AMPK n'est modifiée que par les régimes variant à la fois par leurs teneurs en énergie et en protéines (variation probable du catabolisme des acides aminés comme l'attesteraient les mesures 
d'acide urique), alors que la voie impliquée dans la régulation du métabolisme protéique est principalement affectée par les alternances des régimes différant par leur teneur en protéines. Enfin, d'autres résultats ont montré avec l'alimentation séquentielle des modifications de concentrations plasmatiques en hormones thyroïdiennes régulant le métabolisme énergétique, et d'expression de gènes impliqués dans la lipogenèse et dans l'oxydation des acides gras (Rosebrough et al 1989 et 2011, Joubert et al 2008). Le poulet régule certainement son métabolisme énergétique musculaire et hépatique en fonction des séquences et des apports nutritionnels. Ceci lui permettrait un stockage ou une utilisation rapide des substrats énergétiques en fonction de la composition du régime offert.

Ainsi, il existe en fonction de la dynamique d'apport alimentaire des adaptations métaboliques au niveau de l'utilisation ou du stockage des nutriments (variation de l'équilibre protéosynthèse/ protéolyse, de la lipogenèse et de l'utilisation des nutriments énergétiques). Ces mécanismes de régulation permettent au poulet, dans certaines conditions, de valoriser des régimes déséquilibrés. Ils pourraient aussi représenter un outil pour moduler le métabolisme tissulaire et en conséquence la qualité des produits.

\section{2 / Incidence sur la qualité des produits}

\section{1 / Composition corporelle}

De nombreuses recherches ont été menées afin de mesurer l'impact de différents macronutriments (protéines, acides aminés, énergie...) sur les performances de croissance et sur les rendements en viande des volailles et plus particulièrement des poulets. Il est ainsi clair que le gain de poids et le rendement en filet sont favorisés par une augmentation de l'ingéré en acides aminés alors qu'une restriction de celui-ci entraîne une réduction des poids absolu et relatif du muscle pectoral et augmente la part du gras abdominal (Tesseraud et al 2003).

La lysine est connue pour exercer des effets spécifiques sur la composition des carcasses et la croissance musculaire (Leclercq 1998). Un effet particulièrement drastique a été observé sur le développement du muscle pectoral chez le poulet (Tesseraud et al 1996a). Le muscle pectoral du poulet représente un important « réservoir » de lysine qui peut être mobilisé dans les états de déficience en cet acide aminé. L'accroissement de la protéolyse musculaire dans de telles conditions fournit des acides aminés libres qui sont utilisés pour la synthèse des pro- téines corporelles. Il est à noter que ni la thréonine ni la valine ne présentent un effet sur la composition corporelle aussi prononcé que celui de la lysine, comme observé dans une expérience dans laquelle ces trois acides aminés ont été étudiés séparément dans des conditions similaires (Leclercq 1998). L'effet spécifique de la lysine sur le développement du muscle pectoral peut être dû au fait que la lysine est le plus abondant des acides aminés essentiels dans les protéines du muscle squelettique.

Il semble, de plus, que les acides aminés modifient la composition chimique des tissus. En particulier, une alimentation déficiente en acides aminés soufrés affecte la teneur en lipides et protéines corporelles, mais également la composition en acides aminés au niveau tissulaire chez le poulet (Conde-Aguilera et al 2013) comme chez le porcelet (Conde-Aguilera et al 2010 et 2014).

Au-delà de ces critères zootechniques, les apports nutritionnels (quantitatifs ou qualitatifs) influencent des critères concernant la qualité de la viande de volaille ou sa transformation. Nous détaillerons ici deux aspects de la qualité de la viande de volaille, en lien respectivement avec sa sensibilité à l'oxydation et avec son acidification post-mortem (pH) (figure 3).

\section{2 / Qualité de la viande, en lien avec sa sensibilité à l'oxydation}

De nombreux facteurs nutritionnels sont susceptibles d'avoir une action sur l'oxydation de la viande de volaille : vitamine $\mathrm{E}$, vitamine $\mathrm{C}$, antioxydants naturels ou de synthèse, huiles essentielles, sélénium, qualité et quantité des matières grasses incorporées dans l'aliment, etc. (Tavarez et al 2011). Les niveaux de protéines et d'énergie des aliments ne sont pas mentionnés comme étant des paramètres ayant une influence directe sur la capacité d'oxydation des viandes de volaille. En revanche, comme rappelé dans la partie 1 de cette revue, la méthionine peut jouer un rôle dans la défense contre le stress oxydant (Métayer et al 2008, Tesseraud et al 2009b). Selon la source de méthionine apportée dans le régime (DL-méthionine, DLM ou son hydroxy-analogue, le DL-HMTBA), l'impact sur le stress oxydant et la peroxydation lipidique, y compris dans le muscle post-mortem, sera plus ou moins marqué (Martin-Venegas et al 2006, Swennen et al 2011, Willemsen et al 2011). Ainsi, la substitution partielle (50\%) de DLM par du DL-HMTBA réduit significativement l'oxydation des lipides durant la conservation (mesurée au travers de l'indice TBARS - i.e. substances réactives à l'acide thiobarbiturique indice de la peroxydation lipidique) au niveau du filet mais aussi de la cuisse (Mercier et al 2009, Berri et al 2012).

\section{3 / Qualité de la viande, en lien avec son $\mathbf{p H}$}

Jusqu'à une période récente, peu d'information était disponible quant à l'effet de la nutrition sur les caractéristiques de la viande en lien avec son $\mathrm{pH}$. Une première étude a montré que l'augmentation du niveau de lysine dans l'aliment (audelà des besoins pour la croissance musculaire) améliorait la qualité du filet de poulet en augmentant son $\mathrm{pH}$ ultime

Figure 3. Déterminisme de la qualité technologique de la viande de volaille : évolution du $\mathrm{pH}$ post-mortem, lien avec les réserves en glycogène musculaire et principaux défauts de qualité des viandes (en rouge).

DFD «Dark Firm Dry» : viande foncée, dure et sèche ; PSE «Pale Soft Exudative»: viande pâle, molle et exsudative ; $\mathrm{pH} 15^{\prime}$ : $\mathrm{pH}$ de la viande mesuré 15 minutes après abattage; $\mathrm{pH}$ ultime : $\mathrm{pH}$ mesuré 24 heures après abattage.

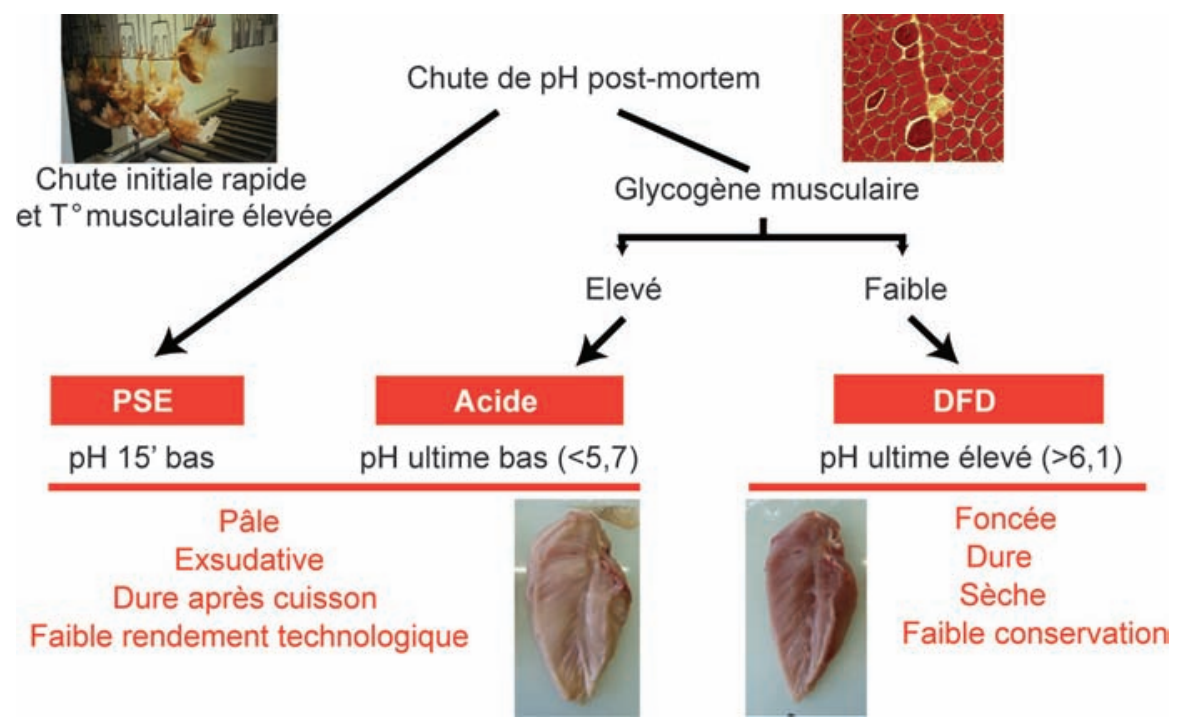


(pHu, mesuré 24 heures après la mort de l'animal) et en diminuant les pertes en eau au cours du stockage (Berri et al 2008). L'utilisation de DL-HMTBA (hydroxy-analogue de la méthionine) peut aussi améliorer de manière significative les propriétés fonctionnelles du filet de poulet via un $\mathrm{pH}$ ultime et des rendements technologiques augmentés. Les mécanismes mis en jeu sont encore à élucider.

Chez le poulet, le $\mathrm{pH}$ ultime constitue un élément-clé dont dépend un grand nombre de caractéristiques technologiques et sensorielles de la viande. Il est essentiellement déterminé par la teneur en glycogène du muscle au moment de la mort de l'animal : plus cette teneur est élevée, plus le $\mathrm{pH}$ final de la viande sera acide et inversement (Le BihanDuval et al 2008). Des résultats récents montrent que les modifications de $\mathrm{pH}$ observées en lien avec des variations d'apport alimentaire passent par des variations de teneurs en glycogène musculaire. Ainsi, le glycogène musculaire est diminué et le $\mathrm{pHu}$ du filet est augmenté lorsque la densité des apports énergétiques et protéiques est augmentée (Zhao et al 2012). Augmenter l'apport en protéines (de 17 à $23 \%$ ) du régime de finition sans modifier l'apport énergétique conduit aussi à une augmentation de la teneur en glycogène du filet avec des conséquences notables en termes de $\mathrm{pH}$, de couleur et de rétention en eau (diminution de 0,1 unité $\mathrm{pH}$ ) (Jlali et al 2012). L'impact de l'aliment sur les caractéristiques du filet peut toutefois dépendre du type génétique, notamment de la capacité d'engraissement des animaux (Jlali et al 2012). D'une manière générale, l'impact de l'apport en protéines sur le $\mathrm{pH}$ et la qualité du filet apparaît comme variable et susceptible de dépendre de nombreux facteurs, liés aux caractéristiques des régimes ou à celles des animaux (Yalçin et al 2010, Lilly et al 2011, Zhao et al 2012).

Dans le but de mieux définir les lois de réponse qui régissent les variations de qualité du filet en fonction de l'apport en acides aminés, une étude récente (Lessire et al 2013) a mis en évidence qu'au-delà de la quantité de protéines, le profil en acides aminés du régime pouvait influencer le $\mathrm{pH}$ ultime de la viande et certains caractères associés (couleur, exsudat). L'objectif initial de l'essai était d'estimer les besoins en acides aminés du poulet de chair pendant la période de finition en faisant varier les niveaux de lysine digestible (de 0,7 à $1,15 \%$ ) et ceux en acides aminés essentiels $(90,100$ et $110 \%$ de la protéine idéale décrite par Mack et al 1999) à énergie constante (Lessire et al 2013).
Les résultats montrent qu'un apport déficitaire en acides aminés (-10\%) conjugué à une teneur réduite en lysine $(0,7 \%)$ favorise la production des viandes avec les $\mathrm{pH}$ les plus élevés $(>6,00)$, les viandes les plus acides $(<5,85)$ étant produites dans le cas d'un apport excédentaire en acides aminés $(+10 \%)$ conjugué à un apport faible en lysine $(0,7 \%)$. Les variations de $\mathrm{pH}$ entre régimes ont eu des conséquences significatives sur la couleur et les pertes par exsudation au cours du stockage.

Connaissant le lien extrêmement étroit qui existe entre $\mathrm{pH}$ ultime et glycogène, il est probable que les changements de $\mathrm{pH}$ induits par les variations d'apports en acides aminés passent par une modulation des réserves énergétiques du muscle. L'hypothèse serait qu'un poulet soumis à un apport en acides aminés excédant ses besoins pour la synthèse protéique et la croissance du muscle utiliserait une partie de ses apports alimentaires à des fins énergétiques (figure 1 et tableau 1), notamment sous forme de glycogène musculaire, expliquant ainsi la diminution de $\mathrm{pH}$ observée. Inversement, réduire les apports en acides aminés pourrait limiter la capacité de stockage du glycogène dans le muscle et expliquer les $\mathrm{pH}$ élevés observés dans ces conditions. La réponse des animaux semble néanmoins dépendante des apports initiaux en lysine (et donc en protéines brutes), qui en modulant la proportion des nutriments utilisés à des fins de synthèse protéique ou à des fins de stockage énergétique vont déterminer les seuils de réponse métabolique des animaux aux variations d'apports en acides aminés.

\section{4 / Vers des stratégies alimen- taires}

Si cette première étude renseigne sur la réponse métabolique des muscles aux variations d'apports en acides aminés (en particulier ceux en lysine), elle ne permet pas d'envisager des solutions concrètes d'amélioration de la qualité des viandes compte-tenu des effets négatifs importants sur la croissance et la composition corporelle des animaux. L'étude présentée dans le paragraphe 1 sur l'alimentation séquentielle (Mameri et al 2010), laisse penser que des stratégies nutritionnelles à court terme pourraient être efficaces pour moduler les réserves énergétiques des muscles et, par conséquent, agir sur le $\mathrm{pH}$ ultime tout en limitant l'impact sur les performances zootechniques. Pour cette raison, des stratégies alimentaires faisant varier l'apport en acides aminés sur une période de 3 jours avant l'abattage ont été évaluées. Elles ont confirmé la possibilité de moduler la qualité des filets en faisant varier l'ap- port en acides aminés, y compris sur une très courte période, avec des conséquences significatives sur le $\mathrm{pH}$ ultime, la couleur et l'exsudat du filet et un impact minime sur les performances et la composition corporelle des animaux (Guardia et al 2014). Des premiers essais ont été réalisés sur le terrain et confirment l'intérêt de ce type de stratégies pour adapter le $\mathrm{pH}$ et le potentiel technologique des filets de poulet en fonction des attentes des industriels (données non publiées).

\section{Conclusion}

Dans le contexte actuel, un objectif majeur en nutrition est le développement de systèmes d'alimentation permettant une production efficace, s'adaptant à la disponibilité en matières premières, et réduisant l'impact environnemental des élevages. Il reste donc crucial d'élaborer des stratégies nutritionnelles qui permettront d'augmenter l'efficacité de transformation des protéines ingérées en protéines musculaires. Ceci suppose des études qui permettent d'identifier des verrous métaboliques sur lesquels il sera possible d'agir et de comprendre la nature dynamique des réponses nutritionnelles (e.g. rythmes d'apports alimentaires, mode de distribution de l'aliment). Il est important de souligner que de nouveaux concepts et techniques, comme par exemple la génomique et la modélisation, sont aussi disponibles pour décrypter des mécanismes qu'il était impossible d'analyser de manière adéquate il y a quelques années, ceci dans l'objectif d'obtenir une meilleure compréhension de la réponse de l'animal à la nutrition. Depuis quelques années, la consommation de volaille se caractérise par une forte segmentation de l'offre, qui donne une part de plus en plus importante aux découpes et aux produits élaborés. Il est donc également impératif de pouvoir maîtriser certaines caractéristiques de la viande (comme le $\mathrm{pH}$ ) qui vont conditionner les rendements technologiques mais aussi la qualité des produits finis. L'optimisation des apports alimentaires nécessite donc la prise en compte de ces nouveaux critères importants pour la compétitivité des filières actuelles.

\section{Remerciements}

Les études présentées à titre d'exemples dans cette synthèse ont reçu le soutien financier de l'INRA, du CASDAR (Programme INNOVOL 2009-2012 réalisé dans le cadre de l'UMT BIRD) et de professionnels de l'alimentation animale (ADISSEO, AJINOMOTO EUROLYSINE S.A.S.). 


\section{Références}

Arnal M.A., Mosoni L., Boirie Y., Houlier M.L., Morin L., Verdier E., Ritz P., Antoine J.M., Prugnaud J., Beaufrère B., Mirand P.P., 1999. Protein pulse feeding improves protein retention in elderly women. Am. J. Clin. Nutr., 69, 1202-1208.

Arnal M.A., Mosoni L., Dardevet D., Ribeyre M.C., Bayle G., Prugnaud J., Patureau Mirand P., 2002. Pulse protein feeding pattern restores stimulation of muscle protein synthesis during the feeding period in old rats. J. Nutr., 132, 1002-1008.

Azzam M.M., Zou X.T., Dong X.Y., Xie P., 2011. Effect of supplemental L-threonine on mucin 2 gene expression and intestine mucosal immune and digestive enzymes activities of laying hens in environments with high temperature and humidity. Poult Sci., 90, 2251-2256.

Baillie A.G., Garlick P.J., 1991. Responses of protein synthesis in different skeletal muscles to fasting and insulin in rats. Am. J. Physiol., 260, E891-E896.

Barnes D.M., Calvert C.C., Klasing K.C., 1995. Methionine deficiency decreases protein accretion and synthesis but not tRNA acylation in muscles of chicks. J. Nutr., 125, 2623-2630.

Berri C., Besnard J., Relandeau C., 2008. Increasing dietary lysine increases final $\mathrm{pH}$ and decreases drip loss of broiler breast meat. Poult. Sci., 87, 480-484.

Berri C., Métayer-Coustard S., Geraert P.A., Mercier Y., Tesseraud S., 2012. Effect of methionine sources and levels on broiler meat quality. In : $24^{\text {th }}$ World Poult. Congr., Salvador - Bahia, Brésil, World's Poult. Sci. J., Sup. 1, 352355.

Boirie Y., Dangin M., Gachon P., Vasson M.P., Maubois J.L., Beaufrère B., 1997. Slow and fast dietary proteins differently modulate postprandial protein accretion. Proc. Natl. Acad. Sci. USA, 94, 14930-14935.

Bouillanne O., Neveux N., Nicolis I., Curis E., Cynober L., Aussel C., 2014. Long-lasting improved amino acid bioavailability associated with protein pulse feeding in hospitalized elderly patients: a randomized controlled trial. Nutrition, 30, 544-550.

Boussaid-Om Ezzine S., Métayer-Coustard S. Collin A., Rideau N., Leterrier C., Bouvarel I., Seiliez I., Tesseraud S., 2012. Regulators of protein metabolism are affected by cyclical nutritional treatments with diets varying in protein and energy content. J. Nutr. Biochem., $23,1467-1473$.

Brun J.M., Basso B., Bernardet M.D., Cornuez A., Sellier N., Leroux S., Lessire M., Pitel F., Morisson M., 2012. Maternal methioninerestricted diet, epigenetics and offspring development: the case of force-fed ducks for 'foie gras' production. In : Colloque SF-DOHaD, Paris, France, 8-9.

Conde-Aguilera J.A., Barea R., Le Floc'h N., Lefaucheur L., van Milgen J., 2010. A sulfur amino acid deficiency changes the amino acid composition of body protein in piglets. Animal, 4, 1349-58.

Conde-Aguilera J.A., Cobo-Ortega C., Tesseraud S., Lessire M., Mercier Y., van Milgen J.,
2013. Changes in body composition in broilers by a sulfur amino acid deficiency during growth Poult. Sci., 92, 1266-1275.

Conde-Aguilera J.A., Cobo-Ortega C., Mercier Y., Tesseraud S., van Milgen J., 2014. The amino acid composition of tissue protein is affected by the total sulfur amino acid supply in growing pigs. Animal, 8, 401-409.

Dardevet D., Rémond D., Peyron M.A., Papet I., Savary-Auzeloux I., Mosoni L., 2012. Muscle wasting and resistance of muscle anabolism: the "anabolic threshold concept" for adapted nutritional strategies during sarcopenia. Sci. World J., 1-6.

Davis T.A., Suryawan A., Orellana R.A. Nguyen H.V., Fiorotto M.L., 2008. Postnatal ontogeny of skeletal muscle protein synthesis in pigs. J. Anim. Sci., 86, E13-E18.

Del Vesco A.P., Gasparino E., Oliveira Neto A.R., Guimarães S.E., Marcato S.M., Voltolini D.M., 2013. Dietary methionine effects on IGF-I and GHR mRNA expression in broilers Genet. Mol. Res., 12, 6414-6423.

Fouad A.M., El-Senousey H.K., Yang X.J., Yao J.H., 2013. Dietary L-arginine supplementation reduces abdominal fat content by modulating lipid metabolism in broiler chickens. Animal, $7,1239-1245$

Gryson C., Walrand S., Giraudet C., Rousset P., Migné C., Bonhomme C., Le Ruyet P., Boirie Y., 2014. "Fast proteins" with a unique essential amino acid content as an optimal nutrition in the elderly: growing evidence. Clin. Nutr., 33 , 642-648.

Guardia S., Lessire M., Corniau A., MétayerCoustard S., Mercerand F., Tesseraud S., Bouvarel I., Berri C., 2014. Short-Term nutritional strategies before slaughter are effective in modulating the final $\mathrm{pH}$ and color of broiler breast meat. Poult. Sci., in press.

Hocquette J.F., Tesseraud S., Cassar-Malek I., Chillard Y., Ortigues-Marty I., 2007. Responses to nutrients in farm animals: implications fo production and quality. Animal, 1, 1297-1313.

Humphrey B.D., Stephensen C.B., Calvert C.C., Klasing K.C., 2006. Lysine deficiency and feed restriction independently alter cationic amino acid transporter expression in chicken (Gallus gallus domesticus). Comp. Biochem. Physiol. A Mol. Integr. Physiol., 143, 218-227.

Jlali M., Gigaud V., Métayer-Coustard S., Sellier N., Tesseraud S., Le Bihan-Duval E., Berri C., 2012. Modulation of glycogen and breast meat processing ability by nutrition in chickens: effect of crude protein level in 2 chicken genotypes. J. Anim. Sci., 90, 447-455.

Joubert R., Tesseraud S., Métayer-Coustard S., Crochet S., Buyse J., Swennen Q., Leterrier C. Bouvarel I, Collin A., 2008, 48h-cycle sequential feeding with diets varying in protein and energy contents alters thyroid axis and energy metabolism in chicken. In : $9^{\text {th }}$ Int. Symp. Avian Endocrinol., Leuven, Belgique, 11-15.

Le Bihan-Duval E., Debut M., Berri C.M., Sellier N., Sante-Lhoutellier V., Jégo Y., Beaumont C., 2008. Chicken meat quality: genetic variability and relationship with growth and muscle characteristics. BMC Genet., 9, 6p.
Leclercq B., 1998. Specific effects of lysine on broiler production: comparison with threonine and valine. Poult. Sci., 77, 118-123.

Lessire M., Primot Y., Corrent E., Fraysse P., Tesseraud S., Berri C., 2013. Lysine supply in finishing broilers: effect on performances and meat quality. $4^{\text {th }}$ Int. Symp. Energy Prot. Metabol. Nutr., Sacramento, USA, EAAP publication, 134, 209-210.

Lilly R.A., Schilling M.W., Silva J.L., Martin J.M., Corzo A., 2011. The effects of dietary amino acid density in broiler feed on carcass characteristics and meat quality. J. Appl. Poult. Res., 20, 56-67.

Mack S., Bercovici D., De Groote G., Leclercq B., Lippens M., Pack M., Schutte J.B., Van Cauwenberghe S., 1999. Ideal amino acid profile and dietary lysine specification for broiler chickens of 20 to 40 days of age. Br. Poult. Sci., 40, 257-265.

Mameri H., Dupont J., Joubert R., Collin A., Crochet S., Cailleau-Audouin E., Tesseraud S. Métayer-Coustard S., 2010. Mechanisms regulating the peripheral utilisation of glucose: involvement of AMPK. $3^{\text {rd }}$ Int. Symp. Energy Prot. Metabol. Nutr., Parma, Italy, EAAP publication, 127, 259-260.

Martin-Venegas R., Geraert P.A., Ferrer R., 2006. Conversion of the Methionine Hydroxy Analogue, DL-2-Hydroxy-(4-Methylthio) butanoic Acid, to Sulfur-Containing Amino Acids in the Chicken Small Intestine. Poult. Sci., 85, 1932-1938.

Mercier Y., Berri C., Baéza E, Bordeau T, Chartrin P., Mercerand F., Geraert P.A., 2009. Improvement of muscle oxidative stability and processing yield in relation with dietary methionine sources. Poult. Sci. Assoc. $98^{\text {th }}$ Ann. Meet. Raleigh, North Carolina, USA, 117

Métayer S., Seiliez I., Collin A., Duchêne S., Mercier Y., Geraert P.A., Tesseraud S., 2008. Mechanisms through which sulfur amino acids control protein metabolism and oxidative status. J. Nutr. Biochem., 19, 207-215.

Métayer-Coustard S., Mameri H., Seiliez I., Crochet S., Crépieux P., Mercier Y., Geraert P.A., Tesseraud S., 2010. Methionine deprivation regulates the S6K1 pathway and protein synthesis in avian QM7 myoblasts without activating the GCN2/eIF2 alpha cascade. J. Nutr., 140, 1539-1545.

Millward D.J., Fereday A., Gibson N.R., Pacy P.J., 1996. Post-prandial protein metabolism. Baillieres Clin. Endocrinol. Metab., 10, 533-549.

Muramatsu T., 1990. Nutrition and whole-body protein turnover in the chicken in relation to mammalian species. Nutr. Res. Rev., 3, 211-228.

Muramatsu T., Salter D.N., Coates M.E., 1985. Protein turnover of breast muscle in germ-free and conventional chicks. Br. J. Nutr., 54, 131-145.

Muramatsu T., Kato M., Tasaki I., Okumura J., 1986. Enhanced whole-body protein synthesis by methionine and arginine supplementation in protein-starved chicks. Br. J. Nutr., 55, 635-641.

Proszkowiec-Weglarz M., Richards M.P., Ramachandran R., McMurtry J.P., 2006. Characterization of the AMP-activated protein 
kinase pathway in chickens. Comp. Biochem. Physiol. B Biochem. Mol. Biol., 143, 92-106.

Reeds P.J., Fuller M.F., 1983. Nutrient intake and protein turnover. Proc. Nutr. Soc., 42, 463471 .

Rideau N., Simon J., Leclercq B., 1986. Further characterization of insulin secretion from the perfused duodenum-pancreas of chicken: a comparison of insulin release in chickens selected for high and low abdominal fat content. Endocrinol., 119, 2635-2641.

Rideau N., Simon J., 1989. L-leucine or its keto acid potentiate but do not initiate insulin release in chicken. Am. J. Physiol., 257, E15-19.

Rosebrough R.W., McMurtry J.P., Steele N.C., 1989. Protein and energy relations in the broiler chicken. Chronic or acute effects of alternating protein or intermittent feeding regimens on broiler lipid metabolism. Br. J. Nutr., 61, 223233.

Rosebrough R.W., Russell B.A., Richards M.P., 2011. Further studies on short-term adaptations in the expression of lipogenic genes in broilers. Comp. Biochem. Physiol. A Mol. Integr. Physiol., 159, 1-6.

Sibut V., Le Bihan-Duval E., Tesseraud S., Godet E., Bordeau T., Cailleau-Audouin E., Chartrin P., Duclos M.J., Berri C., 2008. Adenosine monophosphate-activated protein kinase involved in variations of muscle glycogen and breast meat quality between lean and fat chickens. J. Anim. Sci., 86, 2888-2896.

Sinclair K.D., Allegrucci C., Singh R., Gardner D.S., Sebastian S., Bispham J., Thurston A., Huntley J.F., Rees W.D., Maloney C.A., Lea R.G., Craigon J., McEvoy T.G., Young L.E., 2007. DNA methylation, insulin resistance, and blood pressure in offspring determined by maternal periconceptional $B$ vitamin and methionine status. Proc. Natl. Acad. Sci. USA, 104, 19351-19356.

Swennen Q., Geraert P.A., Mercier Y., Everaert N., Stinckens A., Willemsen H., Li Y., Decuypere E., Buyse J., 2011. Effects of dietary protein content and 2-hydroxy4-methylthiobutanoic acid or dl-methionine supplementation on performance and oxidative status of broiler chickens. Br. J. Nutr., 21, 1-10.

Tavárez M.A., Boler D.D., Bess K.N., Zhao J., Yan F., Dilger A.C., McKeith F.K., Killefer J., 2011. Effect of antioxidant inclusion and oil quality on broiler performance, meat quality, and lipid oxidation. Poult. Sci., 90, 922-930.

Tesseraud S., 1995. Métabolisme protéique chez le poulet en croissance. Effet des protéines alimentaires. INRA Prod. Anim., 8, 197-212.

Tesseraud S., Peresson R., Lopes J., Chagneau A.M., 1996a. Dietary lysine deficiency greatly affects muscle and liver protein turnover in growing chickens. Br. J. Nutr., 75, 853-865.

Tesseraud S., Peresson R., Chagneau A.M., 1996b. Age-related changes of protein turnover in specific tissues of the chick. Poult. Sci., 75, 627-631.

Tesseraud S., Maaa N., Peresson R., Chagneau A.M., 1996c. Relative responses of protein turnover in three different skeletal muscles to dietary lysine deficiency in chicks. Br. Poult. Sci., 37, 641-650.

Tesseraud S., Temim S., Le Bihan-Duval E., Chagneau A.M., 2001. Increased responsiveness to dietary lysine deficiency of pectoralis major muscle protein turnover in broilers selected on breast development. J. Anim. Sci., 79, 927-933.

Tesseraud S., Pym R.A.E., Le Bihan-Duval E. Duclos M.J., 2003. Response of broilers selected on carcass quality to dietary protein supply: live performance, muscle development, and circulating insulin-like growth factors (IGF-I and -II). Poult. Sci., 82, 1011-1016.

Tesseraud S., Abbas M., Duchene S., Bigot K., Vaudin P. Dupont J., 2006. Mechanisms involved in the nutritional regulation of mRNA translation: features of avian model. Nutr. Res. Rev., 19, 104-116.

Tesseraud S., Métayer-Coustard S., Boussaid S., Crochet S., Audouin E., Derouet M., Seiliez I., 2007. Insulin and amino acid availability regulate atrogin-1 in avian QT6 cells. Biochem. Biophys. Res. Commun., 357, 181-186.

Tesseraud S., Bouvarel I., Collin A., Audouin E., Crochet S., Seiliez I., Leterrier C., 2009a. Daily variations in dietary lysine content alter the expression of genes related to proteolysis in chicken Pectoralis major muscle. J. Nutr., 139, 38-43.

Tesseraud S., Métayer Coustard S., Collin A., Seiliez I., 2009b. Role of sulfur amino acids in controlling nutrient metabolism and cell functions: implications for nutrition. Br. J. Nutr., 101, 1132-1139.

Tesseraud S., Everaert N., Boussaid-Om Ezzine S., Collin A., Métayer-Coustard S., Berri C., 2011. Manipulating Tissue Metabolism by Amino Acids. World's Poult. Sci. J., 67, 243-251.

Van den Borne J.J.G.C., Verstegen M.W.A., Alferink S.J.J., Giebels R.M.M., Gerrits W.J.J., 2006a. Effects of feeding frequency and feeding level on nutrient utilization in heavy preruminant calve. J. Dairy Sci., 89, 3578-3586.

Van den Borne J.J.G.C., Verstegen M.W.A., Alferink S.J.J., Van Ass F.H.M., Gerrits W.J.J., $2006 \mathrm{~b}$. Synchronizing the availability of amino acids and glucose decreases fat retention in heavy preruminant calves. J. Nutr., 136, 2181-2187.
Van den Borne J.J., Hocquette J.F., Verstegen M.W., Gerrits W.J., 2007. Whole body and muscle energy metabolism in preruminant calves: effects of nutrient synchrony and physical activity. Br. J. Nutr., 97, 667-675.

Walrand S., Boirie Y., 2005. Optimizing protein intake in aging. Curr. Opin. Clin. Nutr. Metab. Care, 8, 89-94.

Wang J., Wu Z., Li D., Li N., Dindot S.V., Satterfield M.C., Bazer F.W., Wu G., 2012. Nutrition, epigenetics, and metabolic syndrome. Antioxid. Redox Signal. 17, 282-301.

Wang W., Wu Z., Dai Z., Yang Y., Wang J., Wu G., 2013. Glycine metabolism in animals and humans: implications for nutrition and health. Amino Acids. 45, 463-477.

Wen C., Chen X., Chen G.Y., Wu P., Chen Y.P., Zhou Y.M., Wang T. Methionine improves breast muscle growth and alters myogenic gene expression in broilers. J. Anim. Sci., 2014 Feb. 3. [Epub ahead of print] PubMed PMID: 24492548.

Willemsen H., Swennen Q., Everaert N., Geraert P.A., Mercier Y., Stinckens A., Decuypere E., Buyse J., 2011. Effects of dietary supplementation of methionine and its hydroxy analog DL-2-hydroxy-4-methylthiobutanoic acid on growth performance, plasma hormone levels, and the redox status of broiler chickens exposed to high temperatures. Poult. Sci., 90, 2311-2320.

Wilson G.J., Layman D.K., Moulton C.J., Norton L.E., Anthony T.G., Proud C.G., Rupassara S.I., Garlick P.J., 2011. Leucine or carbohydrate supplementation reduces AMPK and eEF2 phosphorylation and extends postprandial muscle protein synthesis in rats. Am. J. Physiol. Endocrinol. Metab., 301, E1236-1242.

Wu G., 2009. Amino acids: metabolism, functions, and nutrition. Amino Acids, 37, 1-17.

Wu G., 2013. Functional amino acids in nutrition and health. Amino Acids, 45, 407-411.

Wu G., Wu Z., Dai Z., Yang Y., Wang W., Liu C., Wang B., Wang J., Yin Y., 2013. Dietary requirements of "nutritionally non-essential amino acids" by animals and humans. Amino Acids, 44, 1107-1113.

Yalçin S., Özkul H., Özkan S., Gous R., Yaşa I., Babacanoğlu E., 2010. Effect of dietary protein regime on meat quality traits and carcase nutrient content of broilers from two commercial genotypes. Br. Poult. Sci., 51, 621-628.

Zhao J.P., Zhao G.P., Jiang R.R., Zheng M.Q., Chen J.L., Liu R.R., Wen J., 2012. Effects of diet-induced differences in growth rate on metabolic, histological, and meat-quality properties of 2 muscles in male chickens of 2 distinct broiler breeds. Poult. Sci., 91, 237-247. 


\title{
Résumé
}

Pour optimiser la composition corporelle et la qualité des viandes tout en garantissant l'efficacité des systèmes de production, il faut comprendre et maîtriser les mécanismes régulant l'utilisation métabolique des nutriments. Dans cette synthèse, nous présentons les généralités concernant la régulation nutritionnelle du métabolisme. Quelques exemples choisis et représentatifs (i.e. apports en protéines et acides aminés, rythme d'apport) sont utilisés pour illustrer ce type de recherche en privilégiant les résultats obtenus lors d'études récentes et en précisant leur intérêt pratique. Nous détaillons leur incidence sur la qualité des produits, et plus généralement l'efficacité des systèmes de production de viande de volaille, au travers d'études impliquant des recherches fondamentales et appliquées. Sont abordés les aspects concernant la composition corporelle et la qualité de la viande en lien avec sa sensibilité à l'oxydation ou avec son pH. Par exemple, chez le poulet, le pH ultime constitue un élément clé dont dépendent plusieurs caractéristiques technologiques et sensorielles de la viande. Il est en grande partie déterminé par la teneur en glycogène du muscle au moment de la mort des animaux, qui dépend entre autres du statut nutritionnel des animaux. Le défi de l'ensemble de ces travaux est de générer des connaissances scientifiques originales, mais aussi de permettre d'optimiser les apports alimentaires (quantitatifs, qualitatifs et rythmes) au cours du développement et en fonction des objectifs de production.

\begin{abstract}
Adapting protein-energy metabolism to optimize poultry carcass and meat quality

To optimize body composition and meat quality while ensuring the efficiency of production systems, it is necessary to understand and control the mechanisms regulating the metabolic utilization of nutrients. In this review, we present an overview on the nutritional regulation of metabolism. Selected and representative examples (i.e. protein and amino acid provision, nutrient delivery) are used to illustrate this type of research by focusing on the results obtained from recent studies and indicating their practical interest. We detail their impact on the quality of products, and more generally the efficiency of production systems for poultry meat, through studies involving basic and applied research. The following issues are discussed: body composition, meat quality in relation to its susceptibility to oxidation or to its $\mathrm{pH}$. For example, in the chicken, the ultimate $\mathrm{pH}$ is a key element, which influences several technological and sensory meat quality traits. It is largely determined by the content of muscle glycogen at slaughter that also depends on the nutritional status of the animals. The challenge for this work is to generate original scientific knowledge but also to optimize dietary supplies (quantitative and qualitative) over time and as a function of production targets.
\end{abstract}

TESSERAUD S., BOUVAREL I., FRAYSSE P., MÉTAYER-COUSTARD S., COLLIN A., LESSIRE M., BERRI C., 2014. Optimiser la composition corporelle et la qualité des viandes de volailles en modulant le métabolisme par les acides aminés alimentaires. INRA Prod. Anim., 27, 337-346. 